\title{
Densidade de fêmeas de Trichospilus diatraeae (Hymenoptera: Eulophidae) por pupa de Diatraea saccharalis (Lepidoptera: Crambidae)
}

\author{
Elizangela Leite Vargas ${ }^{1 *}$, Fabricio Fagundes Pereira ${ }^{2}$, Vanessa Rodrigues Ferreira Calado ${ }^{1}$, Daniele \\ Fabiana Glaeser ${ }^{3}$, Bruna Aparecida Cáceres Rodrigues ${ }^{2} \&$ Nicholas Vinícius Silva $^{2}$ \\ ${ }^{1}$ Faculdade de Ciências Agrárias e ${ }^{2}$ Faculdade de Ciências Biológicas e Ambientais. Universidade Federal da \\ Grande Dourados, Dourados, Mato Grosso do Sul, Brasil. \\ ${ }^{3}$ Embrapa Agropecuária Oeste (EMBRAPA- CPAO), Dourados, Mato Gosso do Sul, Brasil.
}

\begin{abstract}
Resumo - A densidade de fêmeas parasitoides interfere na capacidade de parasitar hospedeiros. O número de fêmeas de Trichospilus diatraeae (Hymenoptera: Eulophidae) por pupa de Diatraea saccharalis (Lepidoptera: Crambidae) foi determinado para criação desse parasitoide em laboratório. Pupas do hospedeiro foram submetidas ao parasitismo por fêmeas de T. diatraeae nas densidades 1:1, 7:1, 14:1, 21:1, 28:1 e 35:1 (parasitoide:hospedeiro). As fêmeas parasitoides e as pupas foram acondicionadas em tubos de vidro $(8,5 \times 2,5 \mathrm{~cm}) \mathrm{e}$ mantidas em contato por $24 \mathrm{~h}$, em câmara climatizada a $25 \pm 2^{\circ} \mathrm{C}, 70 \pm 10 \%$ de umidade relativa e fotofase de $14 \mathrm{~h}$. A porcentagem de parasitismo e emergência de $T$. diatraeae sobre pupas de $D$. saccharalis foi de $100 \%$ em todas as densidades do parasitoide. A duração do ciclo biológico (ovo-adulto) de T. diatraeae foi de 19,25 $\pm 0,22$ dias na densidade de 1:1 e 17,00 $\pm 0,00$ dias na de 35:1. A progênie por pupa variou de $106,00 \pm 12,38$ a 514,67 $\pm 54,55$ descendentes por pupa nas densidades 1:1 e 35:1, respectivamente. A razão sexual diminuiu com o aumento da densidade, variando entre $0,97 \pm 0,01$ e $0,89 \pm 0,01$ nas densidades $1: 1$ e $35: 1$, respectivamente. As porcentagens de parasitismo e emergência de $T$. diatraeae sobre pupas de $D$. saccharalis não foram influenciadas pelas densidades de fêmeas do parasitoide. As densidades de 14 a 21 fêmeas de $T$. diatraeae por pupa de $D$. saccharalis são mais adequadas para criação desse parasitoide em condições de laboratório.
\end{abstract}

Palavras-chave adicionais: controle biológico, criação massal, densidade de parasitoide, parasitoide pupal.

\begin{abstract}
Females density of Trichospilus diatraeae (Hymenoptera: Eulophidae) on pupae of Diatraea saccharalis (Lepidoptera: Crambidae)) - Density of parasitoid females affects their capacity to develop within the host. The number of Trichospilus diatraeae (Hymenoptera: Eulophidae) females per pupa of Diatraea saccharalis (Lepidoptera: Crambidae) was adjusted to study the effect of parasitoid production in the laboratory. Host pupae were parasitised by females of $T$. diatraeae at densities of 1:1, 7:1, 14:1, 21:1, 28:1 and 35:1 (parasitoid:host). The females of the parasitoid remained in contact with pupae for $24 \mathrm{~h}$ in glass tubes $(8.5 \times 2.5 \mathrm{~cm})$, packed in a climatic chamber regulated at $25 \pm 2^{\circ} \mathrm{C}, 70 \pm 10 \%$ relative humidity and light regime of $14 \mathrm{~h}$. The percentage of parasitism and emergence of T. diatraeae on pupae of $D$. saccharalis was $100 \%$ in all parasitoid densities. The length of thr cycle (egg-adult) of Trichospilus diatraeae was $19.25 \pm 0.22$ days at a density of $1: 1$ and $17.00 \pm 0.00$ days at 35:1. The offspring number of $T$. diatraeae was $106.00 \pm 12.38$ and $514.67 \pm 54.55$ individuals per pupa at densities of $1: 1$ and $35: 1$, respectively. The sex ratio decreased with increasing density, ranging between $0.97 \pm 0.01$ and $0.89 \pm 0.01$ at densities of $1: 1$ and 35:1, respectively. The percentages of parasitism and emergence of $T$. diatraeae on pupae of $D$. saccharalis were not affected by the densities of female parasitoids. The densities of 14 to 21 females of $T$. diatraeae per pupa of $D$. saccharalis are suitable for rearing this parasitoid under laboratory conditions.
\end{abstract}

Additional key words: biological control, density of parasitoid, mass rearing, pupal parasitoid.

Trichospilus diatraeae Cherian \& Margabandhu, 1942, foi descrita a partir de exemplares coletados em pupas de Diatraea venosata (Walker, 1863) (Lepidoptera: Crambidae) provenientes do sul da Índia (Cherian \& Margabandhu 1942). Este himenóptero da família Eulophidae é um parasitoide pupal que tem sido estudado como agente potencial no controle biológico de pragas em diversas culturas, como canade-açúcar, milho e algodão, em países da África, Ásia e Américas (Boucek 1976). No Brasil, T. diatraeae foi registrada parasitando naturalmente Cerconota anonella (Sepp., 1830) (Lepidoptera: Oecophoridae) em gravioleira (Oliveira et al. 2001), Thyrinteina arnobia (Stoll, 1782) e Melanolophia consimilaria

Autora para correspondência: elileitevargas@gmail.com

Editor responsável: Freddy Bravo

Submetido: 18 dez. 2012; aceito: 30 jun. 2013

Publicação inicial: 7 ago. 2013; versão final: 2 maio 2014
(Walker, 1860) (Lepidoptera: Geometridae) em plantio de eucalipto (Pereira et al. 2008; Zaché et al. 2010) e, recentemente, Diaphania hyalinata (Linnaeus, 1758) (Lepidoptera: Pyralidae) em plantio de abóbora (Melo et al. 2011). Tendo em vista que a adoção de estratégias complementares para o controle de insetospraga é uma premissa do Manejo Integrado de Pragas, o uso de parasitoides é uma alternativa promissora nessa direção (Goulart et al. 2011).

A falta de sucesso de algumas liberações de parasitoides no controle de pragas pode estar associada à proporção inadequada de fêmeas em relação à densidade de hopedeiros no agroecossistema (Paron et al. 1998), pois o número de fêmeas por hospedeiro afeta a capacidade de parasitismo (Sampaio et al. 2001). A densidade de fêmeas parasitoides interfere na supressão da defesa celular do hospedeiro (Andrade et al. 2010) e, em consequência, na produção da progênie 
(Sagarra et al. 2000). A razão sexual (Zacarin et al. 2004; Irvin \& Hoddle 2006; Carneiro et al. 2009), o tempo de desenvolvimento e a longevidade dos parasitoides (Silva-Torres \& Matthews 2003) também podem ser alteradas com a densidade de parasitoides por hospedeiro.

Trabalhos prévios avaliaram a reprodução de fêmeas de $T$. diatraeae em pupas de Anticarsia gemmatalis Hübner, 1818 (Lepidoptera: Erebidae), T. arnobia, Hylesia paulex Hübner, 1820 (Lepidoptera: Saturniidae) e Tenebrio molitor Linnaeus, 1758 (Coleoptera: Tenebrionidae) (Pastori et al. 2012a,b; Favero et al. 2013). A capacidade reprodutiva de $T$. diatraeae também foi estudada em pupas de D. saccharalis, porém, apenas uma fêmea foi exposta ao hospedeiro (Paron \& Berti-Filho 2000). No entanto, a multiplicação de $T$. diatraeae depende de estudos que determinem a densidade de fêmeas deste parasitoide por pupa de Diatraea saccharalis (Fabricius, 1794) (Lepidoptera: Crambidae), principalmente devido ao seu hábito gregário. Sendo assim, o objetivo deste trabalho foi determinar o número adequado de fêmeas de $T$. diatraeae por pupa de $D$. saccharalis para criação do parasitoide em laboratório.

\section{MATERIAL E MÉtodos}

Local de condução dos experimentos. O experimento foi realizado no Laboratório de Entomologia e Controle Biológico (LECOBIOL) da Faculdade de Ciências Agrárias (FCA) da Universidade Federal da Grande Dourados (UFGD), em Dourados, Mato Grosso do Sul.

Criação de Diatraea saccharalis (Lepidoptera: Crambidae). Pupas de D. saccharalis foram cedidas pela empresa BUG - Agentes Biológicos para o estabelecimento da criação em laboratório. Adultos desse lepidóptero (20 machos e 30 fêmeas) foram mantidos em gaiolas de PVC $(10 \times 22 \mathrm{~cm})$ fechadas com tecido do tipo "voil" e elástico. Essas gaiolas foram revestidas com folhas de papel sulfite umedecido, onde as fêmeas, após o acasalamento, ovipositaram. Após a eclosão, as lagartas de D. saccharalis foram colocadas em potes telados contendo dieta artificial modificada de Hensley \& Hammond (1968), a base de farelo de soja, germe de trigo, vitaminas e sais minerais, onde permaneceram até a formação das pupas. As pupas foram recolhidas, sexadas e colocadas em gaiolas de PVC até a formação dos adultos (Parra 2007).

\section{Criação de Trichospilus diatraeae} (Hymenoptera: Eulophidae). Os adultos de $T$. diatraeae foram mantidos em tubos de vidro $(8,5 \times 2,5 \mathrm{~cm})$ vedados com algodão e alimentados com gotículas de mel puro. Para manutenção da criação, cada pupa de D. saccharalis com 48 h de idade foi exposta a três fêmeas de $T$. howardi por $24 \mathrm{~h}$. Após esse período, as pupas foram individualizadas em tubos de vidro e mantidas em câmara climatizada a $25 \pm 2^{\circ} \mathrm{C}$, $70 \pm 10 \%$ de umidade relativa e fotofase de $14 \mathrm{~h}$, até a emergência de adultos, seguindo a recomendação de Pereira et al. (2008).

Desenvolvimento experimental. Pupas de D. saccharalis com $48 \mathrm{~h}$ foram expostas ao parasitismo por fêmeas de $T$. diatraeae com $24 \mathrm{~h}$ de idade nas densidades $1: 1,7: 1,14: 1,21: 1,28: 1$ e 35:1 (parasitoide : hospedeiro). $\mathrm{O}$ delineamento experimental foi inteiramente casualizado, com 12 repetições para cada tratamento. Foram selecionadas pupas com $0,140 \pm 0,003 \mathrm{~g}$. Após $24 \mathrm{~h}$ em contato com as pupas, as fêmeas de $T$. diatraeae foram retiradas dos tubos de vidro e os hospedeiros individualizados e mantidos em câmara climatizada a $25 \pm 2^{\circ} \mathrm{C}, 70 \pm 10 \%$ de umidade relativa e fotofase de $14 \mathrm{~h}$ até a emergência dos parasitoides adultos. A mortalidade natural do hospedeiro foi calculada utilizando a fórmula de Abbott (1925).

As características biológicas avaliadas foram a porcentagem de pupas parasitadas [(número de pupas de $D$. saccharalis com emergência de parasitoides + pupas sem emergência de adultos de D. saccharalis)/(número total de pupas) $\times 100]$; porcentagem de pupas com emergência de parasitoides [(número de pupas de $D$. saccharalis com emergência de adultos dos parasitoides)/(número de pupas parasitadas) $\times 100$ ]; a progênie (número de parasitoides emergidos por pupa de D. saccharalis); a duração (dias) do ciclo de vida (ovo-adulto); a longevidade média em dias (para avaliação dessa variável, foram selecionados ao acaso 20 fêmeas e 10 machos de T. diatraeae de cada tratamento, sendo esses parasitoides, no dia de sua emergência, individualizados em tubos de ensaio contendo uma gota de mel, onde permaneceram até a sua morte); a largura da cápsula cefálica (para avaliação dessa característica, foram escolhidos ao acaso, em cada tratamento, 15 fêmeas e 15 machos de $T$. diatraeae, visando medir a largura da cápsula cefálica em ocular micrométrica) e a razão sexual $(\mathrm{RS}=$ número de fêmeas/ número de adultos) de $T$. diatraeae. O sexo dos adultos de $T$. diatraeae foi determinado baseado nas características morfológicas da antena e abdome, de acordo com metodologia proposta por Cherian \& Margabandhu (1942).

Os dados da duração do ciclo, do número de parasitoides emergidos por pupa de $D$. saccharalis, da razão sexual e do tamanho da cápsula cefálica de machos e fêmeas de $T$. diatraeae foram submetidos à análise de variância e, quando significativo $(p<0,05)$, foi realizada a análise de regressão. A escolha da equação que melhor se ajustou aos dados foi baseada no coeficiente de determinação $\left(\mathrm{R}^{2}\right)$ e na significância dos coeficientes de regressão $\left(\beta_{\mathrm{i}}\right)$ e da regressão pelo teste $\mathrm{F}(<0,05)$. 


\section{RESULTADOS}

A porcentagem de parasitismo e emergência de $T$. diatraeae sobre pupas de D. saccharalis foi de $100 \%$ nas densidades de fêmeas parasitoides avaliadas; ou seja, de todas as pupas houve emergência de parasitoides.

A duração do ciclo ovo-adulto de $T$. diatraeae em pupas de $D$. saccharalis $(\mathrm{F}=26,4092 ; p=0,0001$; $\left.\mathrm{R}^{2}{ }_{\text {Trat }}=0,5904\right)$ decresceu com o aumento da densidade do parasitoide, com 19,25 $\pm 0,22$ dias na densidade 1:1 e 17,00 $\pm 0,00$ dias na densidade 35:1 (Figura 1). A progênie produzida por pupa de $D$. saccharalis $\left(\mathrm{F}=17,0378 ; p=0,0001 ; \mathrm{R}^{2}{ }_{\text {Trat }}=0,4072\right)$ foi afetada pela densidade de $T$. diatraeae, com médias variando de $106,00 \pm 12,38$ a $514,67 \pm 54,55$ descendentes por pupa nas densidades $1: 1$ e $35: 1$, respectivamente (Figura 2). A progênie produzida por cada fêmea de $T$. diatraeae em pupa de $D$. saccharalis $(\mathrm{F}=71,3292$; $\left.p=0,0001 ; \mathrm{R}_{\text {Trat }}^{2}=0,7054\right)$ declinou com o aumento da densidade e variou entre 103,83 $\pm 12,22$ e $13,08 \pm 1,37$ para as densidades 1:1 e 28:1, respectivamente (Figura 3 ).

A razão sexual de $T$. diatraeae diminuiu com o aumento do número de fêmeas $(\mathrm{F}=16,0011$; $\left.p=0,0001 ; \mathrm{R}_{\text {Trat }}^{2}=0,3400\right)$ e variou de $0,97 \pm 0,01 \mathrm{e}$ $0,89 \pm 0,01$ nas densidades 1:1 e 35:1 (Figura 4). Para a longevidade de fêmeas do parasitoide, não se verificou diferença estatística entre as densidades testadas, com médias de 15,40 $\pm 0,87$ a 19,50 $\pm 0,78$. A longevidade de machos do parasitoide apresentou médias de $15,80 \pm 0,82$ a $18,20 \pm 1,33$, para as densidades testadas, não apresentando diferença estatística.

A largura da cápsula cefálica dos adultos desse parasitoide foi diferente entre os tratamentos. A largura da cápsula cefálica de fêmeas $(\mathrm{F}=90,2861$; $\left.p=0,0001 ; \mathrm{R}_{\text {Trat }}^{2}=0,7894\right)$ e machos $(\mathrm{F}=54,0646$; $\left.p=0,0001 ; \mathrm{R}_{\text {Trat }}^{2}=0,6033\right)$ de $T$. diatraeae variou de

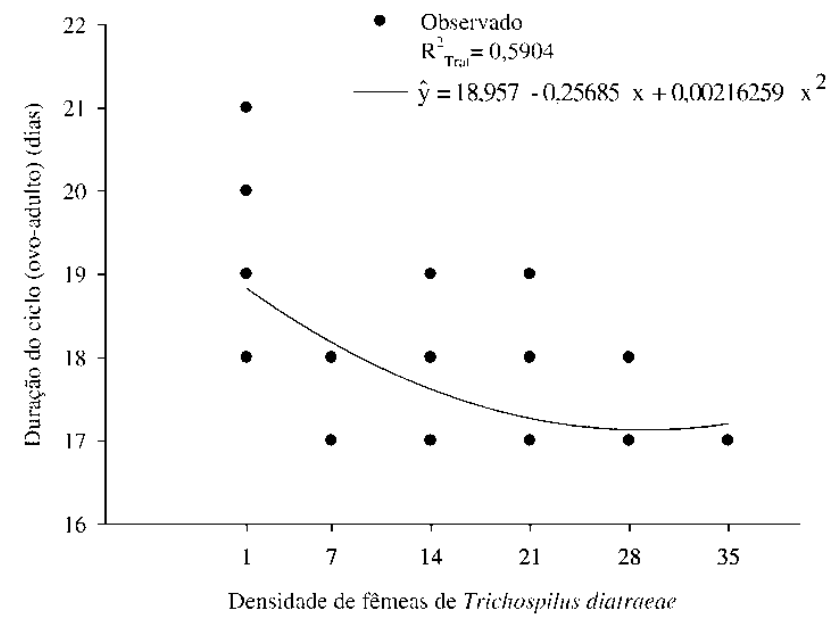

Figura 1. Duração do ciclo (ovo-adulto) de Trichospilus diatraeae em relação ao número de fêmeas por pupa de Diatraea saccharalis a $25 \pm 2{ }^{\circ} \mathrm{C}, 70 \pm 10 \%$ de umidade relativa e fotofase de $14 \mathrm{~h}$ $\left(\mathrm{F}=26,4092 ; p=0,0001 ; \mathrm{R}^{2}{ }_{\text {Trat }}=0,5904\right)$.
$0,360 \pm 0,002 \mathrm{~mm}$ a $0,500 \pm 0,002 \mathrm{~mm}$ e de $0,310 \pm 0,011 \mathrm{~mm}$ a $0,470 \pm 0,006 \mathrm{~mm}$ nas densidades $1: 1$ a $35: 1$, respectivamente (Figura 5 e 6 ).

\section{DISCUSSÃO}

As porcentagens de parasitismo e emergência de $T$. diatraeae sobre pupas de D. saccharalis não foram influenciadas pela densidade de fêmeas do parasitoide e o parasitismo não foi prejudicado mesmo nas altas densidades estudadas. Isto demonstra a adequabilidade ao hospedeiro para o desenvolvimento dos parasitoides, que precisam satisfazer seus requerimentos nutricionais e evitar que o sistema

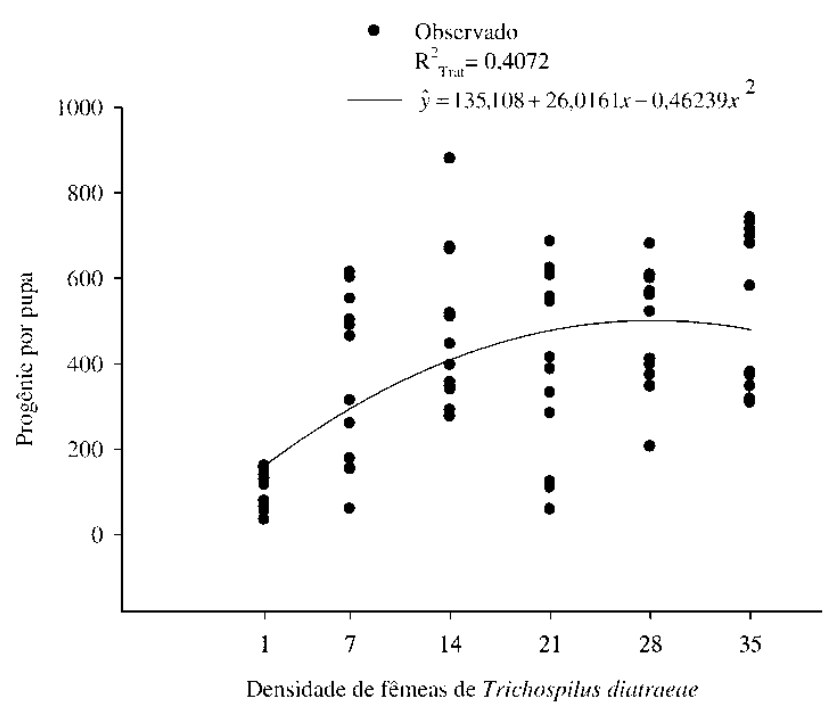

Figura 2. Progênie de Trichospilus diatraeae em relação ao número de fêmeas por pupa de Diatraea saccharalis a $25 \pm 2{ }^{\circ} \mathrm{C}, 70 \pm 10 \%$ de umidade relativa e fotofase de $14 \mathrm{~h}(\mathrm{~F}=17,0378 ; p=0,0001$; $\mathrm{R}_{\text {Trat }}^{2}=0,4072$ ).

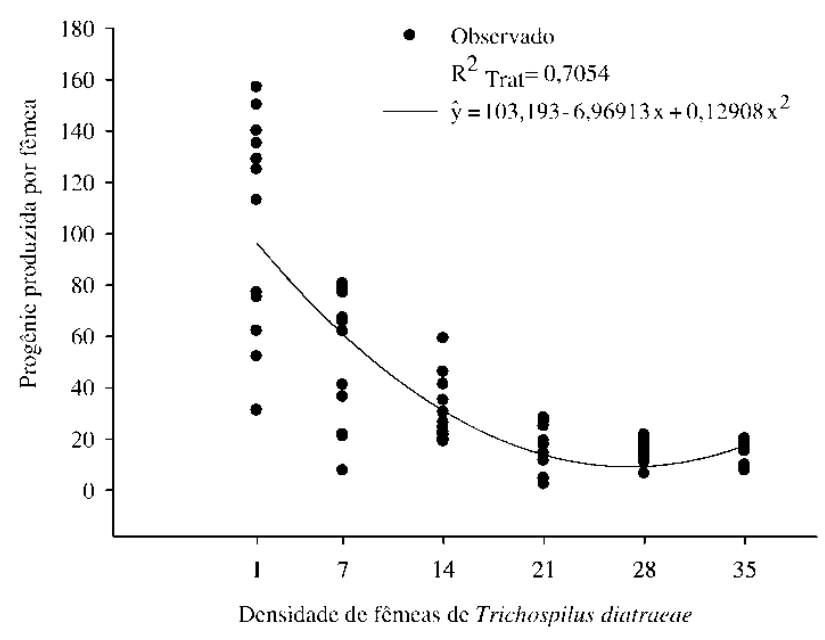

Figura 3. Progênie por fêmea de Trichospilus diatraeae em relação ao número de fêmeas por pupa de Diatraea saccharalis a $25 \pm 2{ }^{\circ} \mathrm{C}$, $70 \pm 10 \%$ de umidade relativa e fotofase de $14 \mathrm{~h}(\mathrm{~F}=71,3292$; $\left.p=0,0001 ; \mathrm{R}^{2}{ }_{\text {Trat }}=0,7054\right)$. 


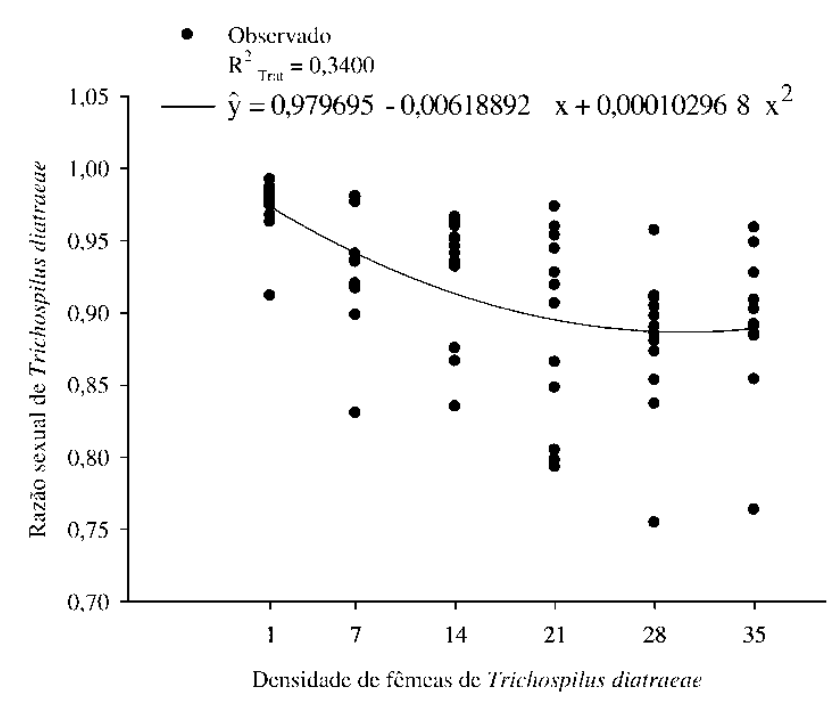

Figura 4. Razão sexual de Trichospilus diatraeae em relação ao número de fêmeas por pupa de Diatraea saccharalis a $25 \pm 2^{\circ} \mathrm{C}, 70$ $\pm 10 \%$ de umidade relativa e fotofase de $14 \mathrm{~h}(\mathrm{~F}=16,0011$; $\left.p=0,0001 ; \mathrm{R}^{2}{ }_{\text {Trat }}=0,3400\right)$.

imunológico do hospedeiro os elimine (Strand \& Pech 1995; Cônsoli \& Vinson 2009). Além disso, maiores densidades de $T$. diatraeae podem reduzir os hemócitos circulantes na hemolinfa do hospedeiro e permitir o desenvolvimento inicial dos seus imaturos, como demonstrado por Andrade et al. (2010) ao multiplicarem $T$. diatraeae em pupas de Anticarsia gemmatalis.

$\mathrm{O}$ aumento da densidade de $T$. diatraeae em pupas de $D$. saccharalis causou decréscimo no ciclo ovoadulto do parasitoide, indicando que a disponibilidade de recurso alimentar pode afetar seu ciclo de vida. O período de desenvolvimento dos imaturos de T. diatraeae em pupas de Tenebrio molitor também diminuiu com o aumento da densidade do parasitoide (Favero et al. 2013). Isto corrobora, também, com o relatado para diferentes densidades de Palmistichus elaiesis Delvare \& LaSalle, 1993 (Hymenoptera: Eulophidae) em pupas de Bombyx mori Linnaeus, 1758 (Pereira et al. 2010) e Melittobia digitata Danhms, 1944 (Hymenoptera: Eulophidae) em pupas de Neobellieria bullata Parker, 1916 (Diptera: Sarcophagidae) (Silva-Torres \& Matthews 2003). A redução do tempo de desenvolvimento de parasitoides em seus hospedeiros pode ser atribuído à competição dos imaturos por nutrientes (Godfray 1994), acelerando a passagem de um instar para o outro.

A progênie de $T$. diatraeae produzida por pupa de D. saccharalis foi afetada pela densidade de fêmeas parasitoides, o que pode estar relacionado com a capacidade de suporte do hospedeiro. A progênie pode variar conforme a espécie hospedeira, como ocorreu com $T$. diatraeae emergidos de pupas de Thyrinteina arnobia, Hylesia paulex (Pastori et al. 2012a) e Spodoptera cosmioides Walker, 1858 (Lepidoptera: Noctuidae) (Zaché et al. 2012). Além disso, a produção

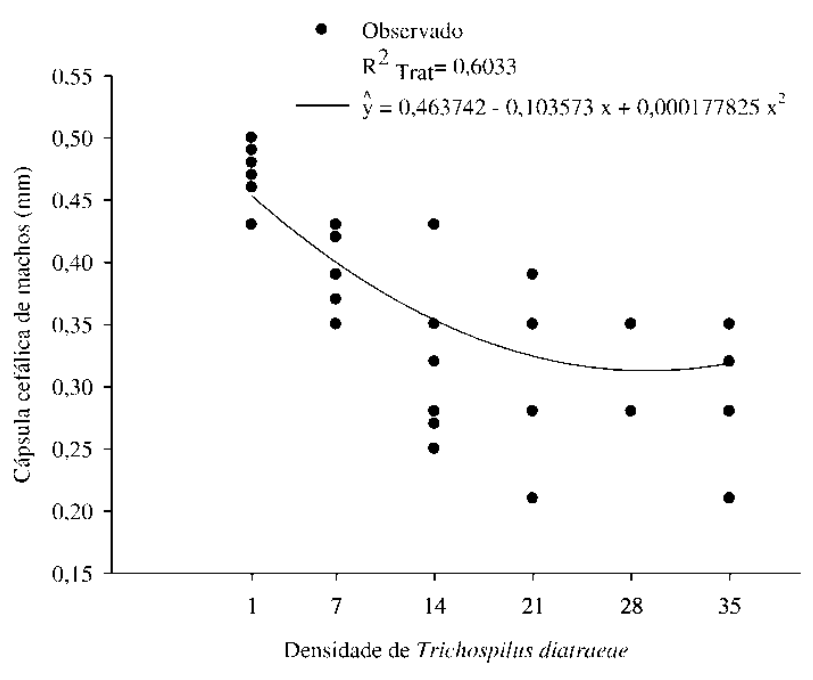

Figura 5. Cápsula cefálica de fêmeas de Trichospilus diatraeae em relação ao número de fêmeas por pupa de Diatraea saccharalis a 25 $\pm 2{ }^{\circ} \mathrm{C}, 70 \pm 10 \%$ de umidade relativa e fotofase de $14 \mathrm{~h}$ $\left(\mathrm{F}=90,2861 ; p=0,0001 ; \mathrm{R}^{2}\right.$ Trat $\left.=0,7894\right)$.

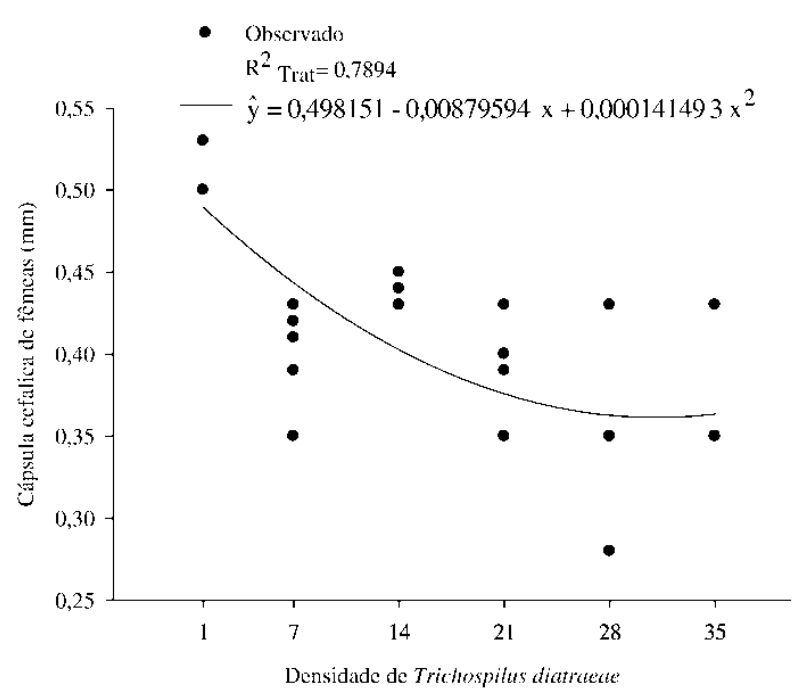

Figura 6. Cápsula cefálica de machos de Trichospilus diatraeae em relação ao número de fêmeas por pupa de Diatraea saccharalis a 25 $\pm 2^{\circ} \mathrm{C}, 70 \pm 10 \%$ de umidade relativa e fotofase de 14 horas $\left(\mathrm{F}=54,0646 ; p=; 0,0001 ; \mathrm{R}^{2}\right.$ Trat $\left.=0,6033\right)$.

de progênie pode depender do grau de competição entre irmãos em desenvolvimento dentro de um hospedeiro compartilhado (Riddick 2008), resultando em menor tamanho corporal (Fidgen et al. 2000). A progênie produzida por cada fêmea de $T$. diatraeae nas pupas de $D$. saccharalis diminuiu o número de indivíduos à medida que aumentou a densidade desse parasitoide. Fêmeas de Anagyrus kamali Moursi, 1948 (Hymenoptera: Encyrtidae) parasitando Maconellicoccus hirsitus Green, 1908 (Hemiptera: Pseudococcidae) apresentaram uma forte tendência de evitar o superparasitismo (Sagarra et al. 2000), regulando o número de ovos colocados no hospedeiro (Hohmann \& Luck 2004). 
O número de fêmeas de $T$. diatraeae produzidas em cada pupa de $D$. saccharalis reduziu com o aumento da densidade do parasitoide. Isso pode ser devido à insuficiência de reservas nutritivas necessárias para o desenvolvimento de fêmeas parasitoides (Sagarra \& Vincent 1999), pois o aumento substancial na descendência masculina pode resultar em intensa competição intraespecífica de larvas (Irvin \& Hoddle 2006). A proporção sexual também pode ser alterada pelo superparsitismo (Riddick 2007), o que pode ser resultado do confinamento de fêmeas parasitoides a um único hospedeiro (Patel et al. 2003) por tempo além daquele necessário para o parasitismo natural.

A razão sexual de parasitoides é influenciada por muitos fatores, tais como o número de parasitoides, densidade e qualidade do hospedeiro (Godfray 1994). Quando apenas uma fêmea parasitoide coloniza um hospedeiro, existe a tendência de que a mesma produza o número de machos suficientes para fertilizar as fêmeas que depositou naquele hospedeiro (Carneiro et al. 2009). Em T. diatraeae, a proporção de machos é muito baixa em relação a fêmeas, porém esse fato não interfere no potencial de fecundação (Bounier 1975). Dessa forma, as proporções sexuais das espécies parasitoides podem ser controladas potencialmente, de preferência em favor das fêmeas, propiciando programas de controle biológico mais efetivos, com predominância de indivíduos do sexo feminino para liberações no campo (Cañete \& Foerster 2003; Zacarin et al. 2004), uma vez que são as fêmeas que parasitam e impedem a emergência do adulto hospedeiro.

A longevidade dos adultos de $T$. diatraeae foi semelhante nas diferentes densidades. Em criações massais de parasitoides, a capacidade de sobrevivência é um dos requisitos para o controle de qualidade (Carneiro et al. 2009). Além disso, a maior longevidade representa uma característica favorável à espécie, pois confere às fêmeas parasitoides maior tempo de busca por hospedeiros no campo em condições de escassez dos mesmos (Foerster et al. 1999).

O tamanho da cápsula cefálica de $T$. diatraeae foi afetado negativamente pela densidade, sendo maior quando se utilizou menores densidades de fêmeas por pupa. Nos parasitoides, fêmeas maiores têm maior potencial reprodutivo do que fêmeas menores (Hohmann \& Luck 2004) e podem viver por mais tempo sem alimento (Ellers et al. 1998); o mesmo pode ocorrer com machos maiores, tendo um maior tempo de vida e com isso um maior sucesso no acasalamento (Sagarra et al. 2001). Também é possível que o tamanho do parasitoide possa influenciar a escolha do hospedeiro, pois uma fêmea pequena pode ser incapaz de dominar um hospedeiro grande e de boa qualidade (Sagarra et al. 2001). O tamanho do corpo dos machos e fêmeas adultos de Anagyrus kamali (Hymenoptera: Encyrtidae) desenvolvidos em Phenacoccus madeirensis Green, 1923 (Hemiptera: Pseudococcidae) foram reduzidos com o aumento na descendência, diminuindo assim, a aptidão reprodutiva dos descendentes (Chong \& Oetting 2007).

$\mathrm{O}$ número de fêmeas de parasitoides por hospedeiro trata-se de um fator importante a ser verificado no controle de qualidade das criações em laboratório e nas liberações massais, pois interfere em aspectos biológicos do parasitoide, como índice de parasitismo, viabilidade, progênie e razão sexual (Carneiro et al. 2009). Trichospilus diatraeae se desenvolveu nas pupas de $D$. saccharalis em todas as densidades avaliadas, mas a escolha do número de fêmeas por pupa para multiplicação do parasitoide será estabelecida de acordo com o objetivo da criação.

Para realização de estudos experimentais com T. diatraeae, uma fêmea por pupa pode ser suficiente, porém para a produção massal, as densidades de 14 a 21 fêmeas por pupa parece mais adequada. Nessas densidades, $T$. diatraeae apresentou excelentes características biológicas: maior tamanho de cápsula cefálica, maior razão sexual e maior número de progênie. Os resultados obtidos quanto ao período de desenvolvimento, fecundidade e longevidade de $T$. diatraeae demonstram que esse parasitoide apresenta potencial para ser utilizado no controle biológico da broca-da-cana-de-açúcar.

\section{Agradecimentos}

Ao Conselho Nacional de Desenvolvimento Científico e Tecnológico (CNPq) e á Fundação de Apoio ao Desenvolvimento do Ensino, Ciência e Tecnologia do Estado de Mato Grosso do Sul (FUNDECT), pelo suporte financeiro.

\section{REFERÊNCIAS}

Abbott, W.S. 1925. A method of computing the effectiveness of a insecticide. Journal of Economic Entomology 18: 265-267.

Andrade, G.S.; Serrão, J.E.; Zanuncio, J.C.; Zanuncio, T.V.; Leite, G.L.D. \& Polanczyk, R.A. 2010. Immunity of an alternative host can be overcome by higher densities of its parasitoids Palmistichus elaeisis and Trichospilus diatraeae. Plos One 5: 1-7.

Boucek, Z. 1976. The African and Asiatic species of Trichospilus and Cotterellia (Hymenoptera, Eulophidae). Bulletin of Entomological Research 65: 669-681.

Bournier, J.P. 1975. Sur la reproduction parthénogénétique de Trichospilus diatraeae Cher. et Margab. (Hymenoptera: Chalcidoidea). Bulletin de la Société Entomologique de France 80: 116-118.

Cañete, C.L. \& Foerster, L.A. 2003. Incidência natural e biologia de Trichogramma atopovirilia Oatman \& Platner, 1983 (Hymenoptera: Trichogrammatidae) em ovos de Anticarsia gemmatalis Hübner, 1818 (Lepidoptera: Noctuidae). Revista Brasileira de Entomologia 47: 201-204.

Carneiro, T.R.; Fernandes, A.O. \& Cruz, I. 2009. Influência da competição intra-específica entre fêmeas e da ausência de 
hospedeiro no parasitismo de Telenomus remus Nixon (Hymenoptera: Scelionidae) sobre ovos de Spodoptera frugiperda (J. E. Smith) (Lepidoptera: Noctuidae). Revista Brasileira de Entomolologia 53: 482-486.

Cherian, M.C. \& Margabandhu, V. 1942. A new species of Trichospilus (Hymenoptera: Chalcidoidea) from south India. Indian Journal of Entomology 4: 101-102.

Chong, J.H. \& Oetting, R.D. 2007. Progeny fitness of the mealybug parasitoid Anagyrus sp. nov. nr. sinope (Hymenoptera: Encyrtidae) as affected by brood size, sex ratio, and host quality. Florida Entomologist 90: 656-664.

Cônsoli, F.L. \& Vinson, S.B. 2009. Parasitoides. In: A.R. Panizzi \& J.R.P. Parra (eds), Bioecologia e Nutrição dos Insetos. Embrapa Informação Tecnológica, Brasília, p. 837-873.

Ellers, J.; van Alphen, J.J.M. \& Sevenster, J.G. 1998. A field study of size-fitness relationships in the parasitoid Asobara tabida. Journal of Animal Ecology 67: 318-324.

Favero, K.; Pereira, F.F.; Kassab, O.; Oliveira, H.N.; Costa, D.P. \& Zanuncio, J.C. 2013. Biological characteristics of Trichospilus diatraeae (Hymenoptera: Eulophidae) progeny are influenced by the number of females exposed per pupa of Tenebrio molitor (Coleoptera: Tenebrionidae). Florida Entomologist 96: 583-589.

Fidgen, J.G.; Eveleigh, E.S. \& Quiring, D.T. 2000. Influence of host size on oviposition behaviour and fitness of Elachertus cacoeciae attacking a low-density population of spruce budworm Choristoneura fumiferana larvae. Ecological Entomology 25: 156-164.

Foerster, L.A.; Doetzer, A.K. \& Avanci, M.R.F. 1999. Capacidade reprodutiva e longevidade de Glyptapanteles muesebecki (Blanchard) (Hymenoptera: Braconidae) parasitando lagartas de Pseudaletia sequax Franclemont (Lepidoptera: Noctuidae). Anais da Sociedade Entomológica do Brasil. 28: 485-490.

Godfray, H.C.J. 1994. Parasitoids, Behavioral and Evolutionary Ecology. Princeton University Press, Princeton.

Goulart, M.M.P.; Bueno, A.F.; Bueno, A.C.O.F. \& Vieira, S.S. 2011. Interaction between Telenomus remus and Trichogramma pretiosum in the management of Spodoptera spp. Revista Brasileira de Entomolologia 55: 121-124.

Hensley, S.D. \& Hammond, A.H. 1968. Laboratory techniques for rearing the sugar cane borer on an artificial diet. Journal Economy Entomology 61: 1742-1743.

Holmann, C.L. \& Luck R.F. 2004. Effect of host availability and egg load in Trichogramma platneri Nagarkatti (Hymenoptera: Trichogrammatidae) and its consequences on progeny quality. Brazilian Archives of Biology and Technology 47: 413-422.

Invin, N.A. \& Hoddle, MS. 2006. The effect of intraspecific competition on progeny sex ratio in Gonatocerus spp. for Homalodisca coagulata egg masses: exonomic implications for mass rearing and biological control. Biological Control 39: $162-170$.

Melo, R.L.; Pratissoli, D.; Polanczyk, R.A.; Tavares, M.; Milanez, A.M. \& Melo, D.F. 2011. Ocorrência de Trichospilus diatraeae (Hym.: Eulophidae) em broca-das-cucurbitáceas, no Brasil. Horticultura Brasileira 29: 228-230.

Oliveira, M.A.S.; Junqueira, N.T.V.; Icuna, I.M.; Alves, R.T.; Oliveira, J.N.S. \& Andrade, G.A. 2001. Incidência de Danos da Broca do Fruto da Graviola no Distrito Federal. Embrapa Cerrados. Planaltina, Brasil. Disponivel em
$<$ http://www.cpac.embrapa.br/download/180/t>; acesso em 21 ago. 2012.

Paron, M.J.F.O.; Ciociola, A.I. \& Cruz, I. 1998. Resposta de Trichogramma atopovirilia Oatman \& Platner (Hymenoptera: Trichogrammatidae) a diferentes densidades de ovos do hospedeiro natural, Helicoverpa zea (Boddie) (Lepidoptera:Noctuidae). Anais da Sociedade Entomológica do Brasil 27: 427-433.

Paron, M.R. \& Berti-Filho, E. 2000. Capacidade reprodutiva de Trichospilus diatraeae (Hymenoptera: Eulophidae) em pupas de diferentes hospedeiros (Lepidoptera). Scientia Agrícola 57: 355-358.

Parra, J.R.P. 2007. Técnicas de Criação de Insetos para Programa de Controle Biológico. 6 ed. ESALQ/FEALQ, Piracicaba.

Pastori, P.L.; Pereira, F.F.; Andrade, G.S.; Silva, R.O.; Zanuncio, J.C. \& Pereira, A.I.A. 2012a. Reproduction of Trichospilus diatraeae (Hymenoptera: Eulophidae) in pupae of two lepidopterans defoliators of eucalypt. Revista Colombiana de Entomologia 38: 91-93.

Pastori, P.L.; Zanuncio, J.C.; Pereira, F.F.; Pratissoli, D.; Cecon, P.R. \& Serrão, J.E. 2012b. Desenvolvimento e exigências térmicas de Trichospilus diatraeae Cherian \& Margabandhu (Hymenoptera: Eulophidae) criado em pupas de Anticarsia gemmatalis (Hübner) (Lepidoptera: Noctuidae). Revista Brasileira de Biociência 10: 79-85.

Patel, K.J.; Chuster, D.J.S. \& Merage, G.H.S. 2003. Density dependent parasitism and host-killing of Liriomyza trifolii (Diptera: Agromyzidae) by Diclyphus intermedius (Hymenoptera: Eulophidae). Florida Entomologist 86: 8-14.

Pereira, F.F.; Zanuncio, J.C.; Tavares, M.T.; Pastori, P.L. \& Jacques, G.C. 2008. New Record of Trichospilus diatraeae (Hymenoptera: Eulophidae) as a parasitoid of the eucalypt defoliator Thyrinteina arnobia (Lepidoptera: Geometridae) in Brazil. Phytoparasitica 36: 304-306.

Pereira, F.F.; Zanuncio, J.C.; Serrão, J.E.; Zanuncio, T.V.; Pratissoli, D. \& Pastori, P.L. 2010. The density of females of Palmistichus elaeisis Delvare and LaSalle (Hymenoptera: Eulophidae) affects their reproductive performance on pupae of Bombyx mori (Lepidoptera: Bombicidae). Anais da Academia Brasileira de Ciência 82: 1-9.

Riddick, E.W. 2007. Influence of honey and maternal age on egg load of lab-cultured Cotesia marginiventris. Biolological Control 52: 613-618.

Riddick, E.W. 2008. Sting frequency and progeny production of lab-cultured Cotesia marginiventris. Biological Control 53: 295-302.

Sagarra, L.A. \& Vincent, C. 1999. Influence of host stage on oviposition, developments, sex ratio, and survival of Anagyrus kamali Moursi (Hymenoptera: Encyrtidae), a parasitoid of the Hibiscus Mealybug, Maconellicoccus hirsutus Green (Homoptera: Pseudococcidae). Biological Control 15: 51-56.

Sagarra, L.A.; Vincent, C. \& Stewart, R.K. 2000. Mutual interference among female Anagyrus kamali Moursi (Hymenoptera: Encyrtidae) and its impact on fecundity, progeny production and sex ratio. Biocontrol Science and Technology 10: 239-244.

Sagarra, L.A.; Vincent, C. \& Stewart, R.K. 2001. Body size as an indicator of parasitoid quality in male and female Anagyrus kamali (Hymenoptera: Encyrtidae). Bulletin of Entomological Research 91: 363-367. 
Sampaio, M.V.; Bueno, V.H.P. \& Maluf, R.P. 2001. Parasitismo de Aphidius colemani Viereck (Hymenoptera: Aphidiidae) em diferentes densidades de Mysus persicae (Sulzer) (Hemiptera: Aphididae). Neotropical Entomology 30: 81-87.

Silva-Torres, C.S.A. \& Matthews, R.W. 2003. Development of Melittobia australica Girault and M. digitata Dahms (Parker) (Hymenoptera: Eulophidae) parasiting Neollieria bullata (Parker) (Diptera: Sarcophagidae) puparia. Neotropical Entomology 32: 645-651.

Strand, M.R. \& Pech, L.L. 1995. Immunological basis for compatibility in parasitoid-host relationships. Annual Review of Entomology 40: 31-56.

Zacarin, G.G.; Gobbi, N. \& Chaud Netto, J. 2004. Capacidade reprodutiva de fêmeas de Apanteles galleriae (Hymenoptera,
Braconidae) em lagartas de Galleria mellonella e Achroia grisella (Lepidoptera, Pyralidae) criadas com dietas diferentes. Iheringia série Zoologia 94: 139-147.

Zaché, B.; Wilcken, C.F.; Dacosta, R.R. \& Soliman, E.P. 2010. Trichospilus diatraeae Cherian \& Margabandhu, 1942 (Hymenoptera: Eulophidae), a new parasitoid of Melanolophia consimilaria (Lepidoptera: Geometridae). Phytoparasitica 38: 355-357.

Zaché, B.; Wilcken, C.F.; Zaché, R.R.C. \& Souza, N.M. 2012. Novo registro de Trichospilus diatraeae Cherian \& Margabandhu, 1942 (Hymenoptera: Eulophidae), como parasitoide de Spodoptera cosmioides Walker, 1858 (Lepidoptera: Noctuidae) no Brasil. Biota Neotropica 12: 319 322. 